\title{
Brain tumors in children with neurofibromatosis: Additional neuropsychological morbidity? ${ }^{1}$
}

\author{
Anne E. De Winter, Bartlett D. Moore, III, ${ }^{2}$ John M. Slopis, Joann L. Ater, \\ and Donna R. Copeland \\ The University of Texas, M.D. Anderson Cancer Center, Houston, TX 77030 (A.E.D., B.D.M., J.M.S., J.L.A., \\ D.R.C.); and The University of Texas-Houston, Medical School, Houston, TX 77030 (J.M.S.)
}

\begin{abstract}
Neurofibromatosis type 1 is a common autosomal dominant genetic disorder associated with numerous physical anomalies and an increased incidence of neuropsychological impairment. Tumors of the CNS occur in approximately $15 \%$ of children with neurofibromatosis, presenting additional risk for cognitive impairment. This study examines the impact of an additional diagnosis of brain tumor on the cognitive profile of children with neurofibromatosis. A comprehensive battery of neuropsychological tests was administered to 149 children with neurofibromatosis. Thirty-six of these children had a codiagnosis of brain tumor. A subset of 36 children with neurofibromatosis alone was matched with the group of children diagnosed with neurofibromatosis and brain tumor. Although mean scores of the neurofibromatosis plus brain tumor group were, in general, lower than those of the neurofibromatosis alone group, these differences were not statistically significant. Children in the neurofibromatosis plus brain tumor group who received cranial irradiation $(n=9)$ demonstrated weaker academic abilities than did children with brain tumor who had not received that treatment. These results suggest that neurofibromatosis is asso-
\end{abstract}

Received 15 January 1999, accepted 17 May 1999.

${ }^{1}$ Supported by grant NS-31950 from the National Institutes of Health, Bethesda, MD, and by the Texas Neurofibromatosis Foundation, Dallas, TX.

${ }^{2}$ Address correspondence and reprint requests to Bartlett D. Moore, III, Ph.D., Division of Pediatrics, Box 87, U.T. M.D. Anderson Cancer Center, 1515 Holcombe Blvd., Houston, TX 77030.

${ }^{3}$ Abbreviations used are as follows: NF, neurofibromatosis-type 1. ciated with impairments in cognitive functioning, but the severity of the problems is not significantly exacerbated by the codiagnosis of a brain tumor unless treatment includes cranial irradiation. Neuro-Oncology 1, 275-281, 1999 (Posted to Neuro-Oncology [serial online], Doc. 99-05, September 7, 1999. URL <neuro-oncology.mc.duke.edu>)

$\mathrm{N}$ $\mathrm{F}^{3}$ is a common autosomal dominant genetic disorder (incidence, 1/3500) characterized by skin pigmentation, iris hamartomas, axillary freckling, and benign and malignant neural tumors (Riccardi, 1981). An estimated $40 \%$ of children with NF have some form of intellectual or behavioral impairment (Riccardi, 1981). Although the frequency of mental retardation (full-scale IQ and adaptive behavior scores $<70$ ) in children with NF is only slightly higher than in the general population (North et al., 1997), learning disabilities and behaviors characteristic of attention-deficit hyperactivity disorder are more common than in the general population (Eliason, 1986; Riccardi, 1982; Stine and Adams, 1989). Impairments in several functional domains have also been reported, most frequently in visual processing, language, and motor coordination (Eldridge et al., 1989; Varnhagen et al., 1988; Wadsby et al., 1989).

Tumors of the CNS occur in approximately $15 \%$ of children with NF (Duffner et al., 1989; Listernick et al., 1989; Mulvihill et al., 1990), presenting another risk factor for neuropsychological impairment. Moore and colleagues (Moore et al., 1994) evaluated the effects of NF with and without a codiagnosis of a brain tumor and reported no statistically significant group differences on standardized tests of intellectual, academic, and cognitive functioning. Given the small sample size in the study $(n=$ 14 for each group), the variability of expression of the NF 
gene, and the range of impairments that may be associated with brain tumors, replication of these findings in a larger sample is necessary. This study examined the neuropsychological functioning in a larger group $(n=149)$ of children with NF; 36 of these patients $(24 \%)$ had also been diagnosed with a brain tumor. In order to clarify the additional impact of a brain tumor on the neuropsychological functioning of children with NF, matched-group analyses were performed comparing children with NF plus a brain tumor with this subset of children without a codiagnosis of tumor. We hypothesized that the mean scores of children with NF plus a brain tumor would be significantly lower than the mean scores of children with NF but without a codiagnosis of brain tumor.

\section{Methods}

\section{Patients}

Over the past 6 years, 236 children and adolescents with NF have been referred to The University of Texas M.D. Anderson Cancer Center for medical diagnosis and clinical management of NF. Referral sources include family practice pediatricians, ophthalmologists, orthopedists, and internists, as well as the Texas Neurofibromatosis Foundation. No child was referred to the clinic specifically for evaluation of a learning disability or cognitive problem. All children are enrolled in a research protocol investigating the neuroanatomical and cognitive features of NF (National Institutes of Health, R01NS-31950). Part of that protocol involves administering a battery of neuropsychological tests. Informed consent was obtained from children and parents in accordance with institutional guidelines.

The individuals included in this study had a diagnosis of NF type 1, based on diagnostic criteria outlined in the National Institutes of Health consensus statement (NIH, 1988). Individuals were excluded from this report who were outside the age range of 6 to 16 years $(n=82)$ and who were not English speaking $(n=5)$, leaving a sample size of 149 . Of these 149, 113 children had a diagnosis of NF alone and 36 had a codiagnosis of brain tumor.

In order to evaluate the additional morbidity presented by a brain tumor, we matched the 36 children with NF plus a brain tumor with 36 children who had NF alone. The groups were matched first on socioeconomic status (Hollingshead, 1975), then on age, and finally on sex to minimize the potential confounds of these factors on neuropsychological test scores. If no exact match was found on a demographic factor, the next closest child was selected and then the next factor was considered. Matching was done without knowledge of the subjects' test scores. Tumor types and locations are presented in Table 1. Of the 36 children with NF plus a brain tumor, 30 were diagnosed with an optic glioma, 5 were diagnosed with astrocytoma, and 1 was diagnosed with a brainstem glioma. Eight of these 36 children had been treated with surgery, 9 with cranial irradiation, and 1 with chemotherapy prior to neuropsychological evaluation. For those children who received radiation, the neuropsychological assessment was completed, on average, 9.5 months (SD, 4.7 months) after beginning radiation treatment.

\section{Procedures}

Study participants were evaluated with a comprehensive battery of intelligence, academic achievement, and neuropsychological tests. Tests were of short duration and possessed differing task demands to engage the child and maximize attention. Testing was performed in a consultation room by a trained psychometrician and, under average conditions, the complete battery generally required 3 to $4 \mathrm{~h}$ including rest breaks. The neuropsychological test battery is designed to cover a wide range of abilities with added emphasis on cognitive areas, the areas at risk in children with NF. The individual tests that compose the battery have been used extensively in neuropsychological research on children and have been found to be reliable over the age range to be included in this study. Most of the battery has been administered to over 1000 children at the University of Texas M.D. Anderson Cancer Center, and results have been reported in studies of patients with pediatric brain tumors, other pediatric cancers, and NF.

Overall intellectual functioning was estimated using one of four measures. The Wechsler Intelligence Scale for Children-Revised (WISC-R) (Wechsler, 1974) was administered to 52 children, and the Wechsler Intelligence Scale for Children-Third Edition (WISC-III) (Wechsler, 1991) was administered to 94 children. The McCarthy Scales of Children's Abilities (McCarthy, 1972) and the StanfordBinet Intelligence Scale-Fourth Edition (Thorndike et al., 1986) were administered to one child each. One child received the neuropsychological test battery but did not receive an assessment of intellectual functioning. The scores of all intelligence tests were mathematically equated to a normative mean of 100 and an SD of 15 .

Table 1. Frequency of tumor diagnosis by location in children with neurofibromatosis plus a brain tumor

\begin{tabular}{|c|c|c|c|}
\hline \multirow[b]{2}{*}{ Tumor location } & \multicolumn{3}{|c|}{ Tumor diagnosis } \\
\hline & $\begin{array}{l}\text { Optic } \\
\text { glioma }\end{array}$ & $\begin{array}{l}\text { Astro- } \\
\text { cytoma }\end{array}$ & Glioma \\
\hline Optic chiasm & 3 & & \\
\hline Optic chiasm, unilateral nerve & 9 & & \\
\hline Optic chiasm, unilateral tract & 2 & & \\
\hline Optic chiasm, bilateral nerves & 6 & & \\
\hline Unilateral optic nerve & 6 & & \\
\hline $\begin{array}{l}\text { Optic chiasm, bilateral nerves, } \\
\text { bilateral tracts }\end{array}$ & 2 & & \\
\hline $\begin{array}{l}\text { Optic chiasm, unilateral optic nerve, } \\
\text { hypothalamus }\end{array}$ & 1 & & \\
\hline $\begin{array}{l}\text { Optic chiasm, } 3^{\text {rd }} \text { ventricle, } \\
\text { hypothalamus }\end{array}$ & 1 & & \\
\hline Frontal lobe, lateral ventricle & & 1 & \\
\hline Hypothalamus & & 1 & \\
\hline Foramen of Monroe & & $1^{\mathrm{a}}$ & \\
\hline Cerebellum & & 1 & \\
\hline Pons & & 1 & \\
\hline Brainstem & & & 1 \\
\hline
\end{tabular}

Data are for 36 children.

${ }^{\text {a}}$ Subject had an optic glioma of the right optic nerve in addition to an astrocytoma. 
Table 2. Composition of cognitive domains

Domain

Intelligence

Academic achievement

Language

Memory

Visual-motor functions

Visual-perceptual functions

Motor functions

Attention
Tests

Wechsler Intelligence Scale for Children-Revised (Wechsler, 1974) or Wechsler Intelligence Scale for Children-Third Edition (Wechsler, 1991), or Stanford-Binet Intelligence Scale-Fourth Edition (Thorndike et al., 1986), or McCarthy Scales of Children's Abilities (McCarthy, 1972)

Wide Range Achievement Test-Revised (Jastak and Wilkinson, 1984) (spelling and arithmetic subtests), Woodcock Johnson Psycho-Educational Battery-Revised (Woodcock and Johnson, 1989) (math and reading clusters)

Verbal fluency: The F-A-S Test (Gaddes and Crockett, 1975), Rapid Automatized Naming (RAN) (Denckla and Rudel, 1974), Test of Auditory Analysis Skills (TAAS) (Rosner and Simon, 1971)

Verbal Selective Reminding Test (Buschke, 1974), Nonverbal Selective Reminding Test (Fletcher, 1985)

Beery Visual-Motor Integration Test (Beery and Butenika, 1967), Block Design subtest from Wechsler Intelligence Scale for Children-Revised (Wechsler, 1974) or Wechsler Intelligence Scale for ChildrenThird Edition (Wechsler, 1991)

Recognition-Discrimination (Satz and Fletcher, 1982), Judgment of Line Orientation Test (Lindgren and Benton, 1980)

Finger Tapping and Grooved Pegboard (Reitan, 1969)

Trail Making Test-Parts A and B (Reitan, 1969), Freedom from Distractibility Deviation Quotient (arithmetic, digit span, and coding subtests of the Wechsler Intelligence Scale for Children-Third Edition [Wechsler, 1991])
To minimize the variable-to-subject ratio, we grouped individual tests into conceptualized domains representing intelligence, academic achievement, language, memory, visual-motor, visual-perceptual, fine motor, and attention (Table 2).

\section{Statistical Analyses}

Analyses were conducted in two stages. The initial step in data analysis was to describe the general nature of the cognitive deficits in children with NF type 1 by examining the mean scores of each general cognitive domain, as well as the individual tests comprising each domain, in the NF-alone group $(n=113)$. Scores on the intellectual and academic achievement domains were standardized to a mean of 100 and SD of 15 . Scores on the language, memory, visual-motor, visual-perceptual, motor, and attention domains were standardized to a mean of 10 , $\mathrm{SD}$ of 3 . To evaluate the nature, severity, and prevalence of learning impairments, we calculated score discrepancies for intellectual-academic achievement.

The second step in data analysis was to conduct matched-group analyses to quantify differences between the NF-alone and NF-plus-brain-tumor groups. Continuous variables were analyzed with the Student $t$ test; frequency data were compared with chi square analyses. The Fisher exact test was used when appropriate. A $P$ value of 0.005 was adopted for statistical significance to control for multiple group comparisons.

\section{Results}

\section{General Neuropsychological Status of Children with Neurofibromatosis Alone}

In the larger group of children with NF alone $(n=113)$, ages ranged from 6.1 to 15.9 years (mean, 11.1 years; $\mathrm{SD}, 2.7$ years). Socioeconomic status ranged from 1 (high) to 5 (low), with a mean in the middle- to lower- middle class range (mean, 3.4; SD, 1.2). There were 54 boys and 59 girls in this group. There were 72 whites, 21 blacks, and 20 English-speaking Hispanics in the sample.

Within this group, full-scale IQ scores ranged from 56 to 126 with a group mean of 92.7 (SD, 16.6). Nine of the children $(8 \%)$ obtained a full-scale IQ score of 69 or below. Verbal IQs (mean, 92.4; SD, 16.5) were slightly lower than performance IQs (mean, 94.0; SD, 17.3).

Mean scores for the language, memory, visual-motor, motor, and attention domains were also below average, but were within $1 \mathrm{SD}$ of the normative means. The mean group score for the visual-perceptual domain was significantly below the normative mean (see Table 3) and was also considerably lower than the mean score for the

Table 3. Domain scores for children with neurofibromatosis alone

\begin{tabular}{lcc} 
Domain & Mean score ${ }^{\mathrm{a}}$ & $\begin{array}{c}\text { Percent in severely } \\
\text { impaired range }^{\mathrm{b}}\end{array}$ \\
\hline Intellectual & $92.7(16.6)$ & $8.4 \%$ \\
$\quad$ Full-scale IQ & $92.4(16.5)$ & $7.4 \%$ \\
$\quad$ Verbal IQ & $94.0(17.3)$ & $8.3 \%$ \\
$\quad$ Performance IQ & $87.2(17.0)$ & $42.7 \%$ \\
Academic achievement & $91.3(17.4)$ & $16.3 \%$ \\
$\quad$ WRAT-reading & $85.4(18.4)$ & $21.2 \%$ \\
WRAT-math & $86.0(16.3)$ & $16.8 \%$ \\
$\quad$ WRAT-spelling & $90.3(19.4)$ & $17.5 \%$ \\
$\quad$ Woodcock-math & $91.3(17.4)$ & $16.3 \%$ \\
$\quad$ Woodcock-reading & $8.6(3.0)$ & $10.3 \%$ \\
Language & $8.3(3.3)$ & $9.5 \%$ \\
Memory & $7.9(2.8)$ & $9.2 \%$ \\
Visual-motor & $5.9(4.0)$ & $37.5 \%$ \\
Visual-perceptual & $9.3(3.7)$ & $10.4 \%$ \\
Motor & $8.1(2.9)$ & $10.0 \%$ \\
Attention &
\end{tabular}

${ }^{\mathrm{a}}$ Data are means and (SD) for 113 children with neurofibromatosis alone.

${ }^{b}$ Severely impaired is defined as score $\leq 2$ SD below the normative means. 


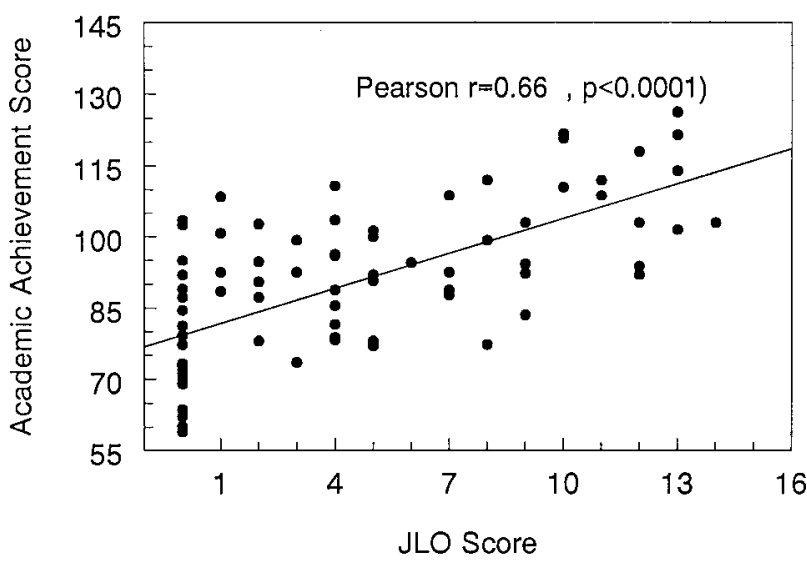

Fig. 1. Association between Judgment of Line Orientation Test (JLO) score and academic achievement score. JLO scores are based on a mean of 10 (SD of 3). Academic achievement scores are based on a mean of 100 (SD of 15).

visual-motor domain. For most of the cognitive domains and for the intelligence scores, the percentage of subjects with scores in the severely impaired range $(2$ or more SDs below average) was about $10 \%$. However, relatively greater numbers of subjects had scores in the severely impaired range in the visual-perceptual domain $(37.5 \%)$. The visual-perceptual domain is comprised of two motor-free measures of visual-spatial functioning, the Recognition Discrimination Test (Satz and Fletcher, 1982), and the Judgement of Line Orientation Test (Lindgren and Benton, 1980). Performance on both of these measures was found to be highly correlated $(P<$ .001) with academic achievement scores in this group of children. As illustrated in Fig. 1, better subtest performance was associated with stronger performance in the academic achievement domain.

The mean score on the academic achievement domain (mean, 87.2; SD, 17.0) was nearly 1 SD below the population mean, indicating that the group as a whole had relatively weak academic achievement skills. As illustrated in Table 3, the performance of approximately $43 \%$ of the sample was in the severely impaired range for academic achievement (2 SD or more below the normative expectation). Within this domain, arithmetic skills appeared affected to a greater degree (WRAT-3, arithmetic: $21.2 \%$ of subjects scored in the severely impaired range; Woodcock, math: $17.5 \%$ scored in the severely impaired range) than spelling or reading abilities (WRAT-3, spelling: $16.8 \%$ scored in the severely impaired range; WRAT-3, reading: $16.3 \%$ scored in the severely impaired range; Woodcock, reading: $8.7 \%$ scored in the severely impaired range).

Intellectual-academic achievement discrepancy scores were also calculated for each child. The mean intellectual-academic achievement domain discrepancy was 4.9 points (see Table 4). Discrepancy scores were also calculated for each measure included in the academic domain. Mean intellectual-academic discrepancies ranged from 1.4 points (Woodcock-Johnson, reading cluster) to 8.2 points (WRAT-3, arithmetic). Discrepancies were statistically significant for the general academic achievement domain $(P=0.0001)$, the WRAT-3 spelling and arithmetic subtests $(P=0.0001)$, and the Woodcock-Johnson math cluster $(P=0.019)$, but not the reading cluster.

\section{Matched-Group Analyses}

In order to assess the impact that a brain tumor diagnosis might have on neuropsychological status, 36 of the subjects with NF alone were matched as closely as possible to the 36 subjects with NF plus a brain tumor, first on socioeconomic status, then on age, and finally on sex. Although subject-by-subject matching was not perfect in all cases, it was successful in forming two groups that did not differ significantly in socioeconomic status, age, or gender.

In general, mean domain scores for children in the NF-plus-brain-tumor group remained within normal limits (within $1 \mathrm{SD}$ of normative values), although there was a consistent trend for these children to perform more poorly than children with NF alone in all domains except intelligence (Table 5). When group mean scores were compared with $t$ tests, however, there were no significant differences in performance between the groups for any domain.

In order to clarify the results of the matched-group analyses, we compared the mean domain scores of children in the NF-plus-brain-tumor group who had received cranial irradiation $(n=9)$ with the children who had not received cranial irradiation $(n=27)$. Scores for children in the irradiation treatment group were consistently lower than scores for children in the no-irradiation group, but these differences were significant only for the academic achievement domain $\left(t_{33}=2.3, P<.05\right)$ (see Table 6).

\section{Discussion}

These results are consistent with other studies suggesting that the diagnosis of NF is associated with relatively lower intellectual, academic, memory, visual-motor, and attention abilities than either the general population (Eliason 1986; Legius et al., 1994; Stine and Adams 1989) or sibling controls (Denckla et al., 1996; Eldridge et al., 1989; Hofman et al., 1994; Mazzocco et al., 1997). By far the greatest impairments were in academic achievement and

Table 4. Intellectual-academic achievement discrepancy scores for children with neurofibromatosis alone

\begin{tabular}{llc} 
& \multicolumn{2}{c}{ Discrepancy score ${ }^{\mathrm{a}}$} \\
\cline { 2 - 3 } Measure & Mean score $^{\mathrm{a}}$ & Significance \\
\hline Academic achievement domain & $4.98(11.11)$ & 0.0001 \\
WRAT-3 & & \\
$\quad$ Spelling & $6.83(13.24)$ & 0.0001 \\
$\quad$ Arithmetic & $8.22(13.99)$ & 0.0001 \\
Woodcock-Johnson & & \\
$\quad$ Math cluster & $2.90(12.28)$ & 0.018 \\
$\quad$ Reading cluster & $1.48(12.19)$ & 0.22 \\
\hline
\end{tabular}

${ }^{\mathrm{a} D}$ Data are mean and (SD) for 113 children with neurofibromatosis alone. 
Table 5. Matched-group analysis

\begin{tabular}{lccc} 
Domain & Mean score for NF alone ${ }^{\mathrm{a}}$ & Mean score for NF $+\mathrm{BT}^{\mathrm{b}}$ & Significance \\
\hline Intellectual & & & \\
$\quad$ Full-scale IQ & $91.7(14.3)$ & $92.9(15.3)$ & $\mathrm{NS}$ \\
$\quad$ Verbal IQ & $92.6(14.6)$ & $91.3(15.1)$ & $\mathrm{NS}$ \\
$\quad$ Performance IQ & $92.2(13.9)$ & $96.3(16.6)$ & $\mathrm{NS}$ \\
Academic achievement & $89.3(13.5)$ & $86.1(14.8)$ & $\mathrm{NS}$ \\
Language & $9.1(3.0)$ & $8.8(2.8)$ & $\mathrm{NS}$ \\
Memory & $8.4(3.1)$ & $7.1(2.8)$ & $\mathrm{NS}$ \\
Visual-motor & $7.9(2.5)$ & $7.3(2.6)$ & $\mathrm{NS}$ \\
Visual-perceptual & $6.1(3.9)$ & $5.8(2.7)$ & $\mathrm{NS}$ \\
Motor & $9.1(3.9)$ & $8.1(3.3)$ & $\mathrm{NS}$ \\
Attention & $8.2(2.3)$ & $7.9(2.3)$ & $\mathrm{N}$ \\
\hline
\end{tabular}

Abbreviations: NS, not significant.

${ }^{a}$ Data are means and (SD) for 36 children with neurofibromatosis alone.

${ }^{b}$ Data are means and (SD) for 36 children with neurofibromatosis plus a brain tumor.

in motor-free visual-spatial abilities. Interestingly, in visual-perceptual tasks requiring a greater degree of motor input (i.e., the visual-motor domain), group mean scores were only slightly impaired relative to the impairments in the pure (motor-free) visual-spatial tasks. Impaired performance on the Judgement of Line Orientation Test (Lindgren and Benton, 1980) continues to be almost a hallmark feature of NF. In this sample of children with NF alone, $58 \%$ had significant deficits (2 or more SDs below average) on this test. In addition, there was a high degree of association between scores on this measure of motor-free visual spatial ability and all subtests of academic achievement (see Fig. 1).

Brain tumors are diagnosed in approximately $15 \%-20 \%$ of children with NF (Duffner et al., 1989; Listernick et al., 1989), and the majority of these tumors are optic gliomas (see Fig. 2). Very little is known regarding the nature of these lesions or their similarity to optic gliomas in non-NF patients. In some cases, the areas of abnormality change very little over time or may resolve spontaneously, leading to the conclusion that they are benign and relatively static lesions such as hamartomas. In other cases, the abnormalities behave more aggressively requiring surgery, chemotherapy, and cranial irradiation therapy (Listernick et al., 1992; Listernick et al., 1994; Roos and Dunn, 1992). For many NF patients with only radiographic findings, careful observation is the treatment of choice unless there is progression of tumor or visual abnormality (Cohen et al., 1990; Roos and Dunn, 1992).

Although no statistically significant differences were found between the groups, there was a consistent trend for those with NF plus a brain tumor to score slightly lower than those with NF alone. Studies of children who have a brain tumor but not NF and who are assessed before chemotherapy or cranial irradiation suggest that having a brain tumor does not by itself mean that a child will have significant neuropsychological deficits unless cranial irradiation is included as part of the treatment regimen (Brookshire et al., 1990; Copeland and Moore, 1992).

The structural and functional effects of cranial irradiation, including periventricular white matter damage, vas- cular alterations, and necrosis, have been documented in numerous studies (Crossan et al., 1994; Packer et al., 1987; Wright and Bresnan, 1976) and are implicated in cognitive decline (Copeland et al., 1985; Copeland et al., 1988; Fletcher and Copeland, 1988; Leiner et al., 1991; Ris and Noll, 1994). We found that the mean domain scores for children in the irradiation treatment group were consistently lower than the scores of children who had not received cranial irradiation. However, only the difference in the academic achievement domain was statistically significant $(P=0.02)$. The small and unequal numbers in the two groups may have prevented significant differences in the other domains from being seen. The mean length of time between the initiation of radiation treatment and neuropsychological assessment was 9.5 months. Although it is possible that posttreatment fatigue and general malaise may have negatively affected these subjects' performances, it is unlikely that significant white matter changes characteristic of delayed radiation effects had developed. The relatively brief period of time between treatment and assessment suggests that tumor-related factors, such as lesion size and rate of growth, might have also hindered the performance of these subjects.

Table 6. Domain scores for brain tumor patients who received radiation versus brain tumor patients who did not receive radiation

\begin{tabular}{lccc} 
Domain & $+\mathrm{XRT}^{\mathrm{a}}$ & $-\mathrm{XRT}^{\mathrm{b}}$ & Significance \\
\hline Intellectual (FSIQ) & $85.2(18.9)$ & $95.5(13.3)$ & $\mathrm{NS}$ \\
Academic achievement & $76.9(18.6)$ & $89.5(12.4)$ & 02 \\
Language & $8.6(4.7)$ & $9.0(2.2)$ & $\mathrm{NS}$ \\
Memory & $5.3(2.8)$ & $7.5(2.7)$ & $\mathrm{NS}$ \\
Visual-motor & $6.2(2.7)$ & $7.9(2.3)$ & $\mathrm{NS}$ \\
Visual-perceptual & $5.4(3.6)$ & $6.0(2.5)$ & $\mathrm{NS}$ \\
Motor & $6.9(3.5)$ & $8.5(3.2)$ & $\mathrm{NS}$ \\
Attention & $7.3(2.8)$ & $8.3(2.1)$ & $\mathrm{NS}$ \\
\hline
\end{tabular}

Abbreviations: FSIQ, full-scale IQ; NS, not significant.

${ }^{a}$ Data are means and (SD) for 9 brain tumor patients who received radiation.

${ }^{b}$ Data are means and (SD) for 27 brain tumor patients who did not receive radiation. 

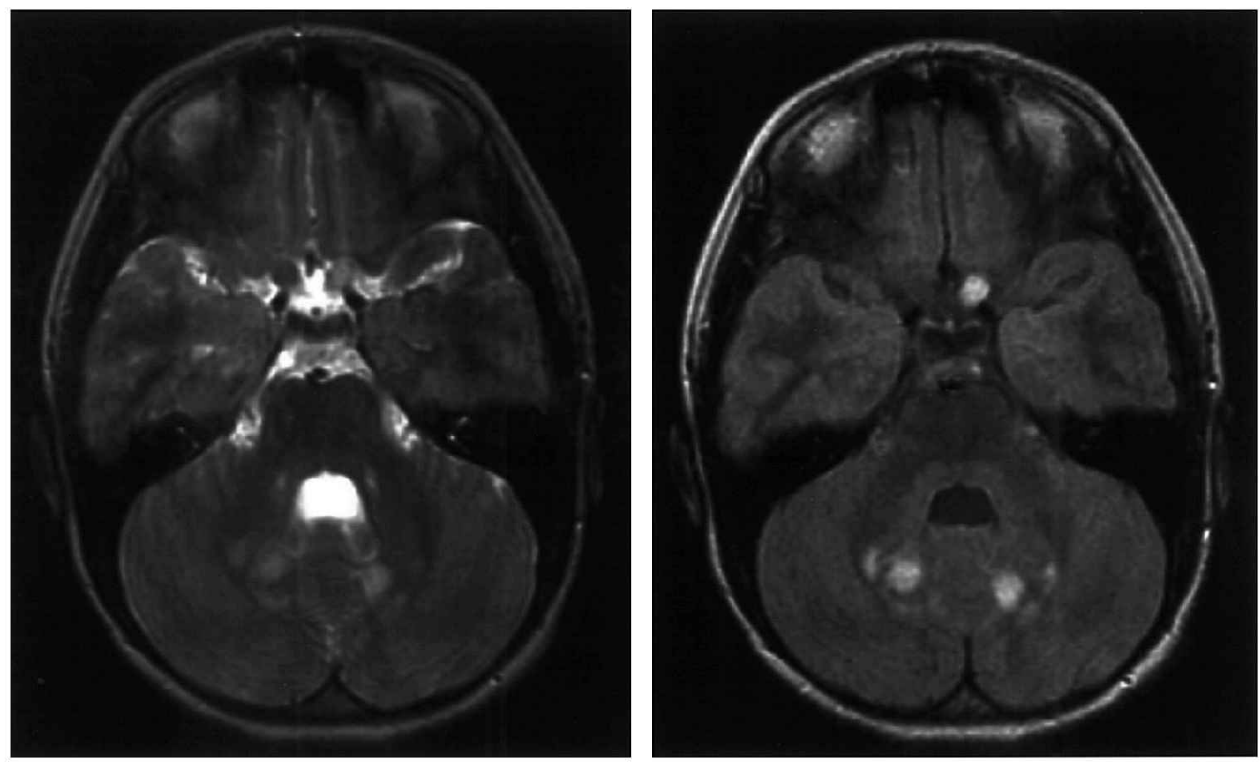

Fig. 2. T-2 v

Neuroimaging studies have revealed areas of abnormality in $50 \%-60 \%$ of NF patients examined (Cohen et al., 1986; Duffner et al., 1989). These abnormalities, which appear as areas of increased signal intensity on T2weighted or fluid attenuated inversion recovery (FLAIR) MRI (see Fig. 2), probably represent "abnormal brain parenchyma, either hamartomas, heterotopias, or local areas of brain dysplasia" (Duffner et al., 1989, page 373). Several studies have examined the relationship of these heterotopias to cognitive functioning in children with NF, but no general consensus has been reached (Denckla et al., 1996; Duffner et al., 1989; Ferner et al., 1993; Hofman et al., 1994; Joy et al., 1995; Moore et al., 1996; North et al., 1994).

In the current study, MRI data available on 77 of the 113 subjects with NF alone showed areas of increased signal intensity in $68 \%$ of the children. Among those with NF plus brain tumor, $85 \%$ evidenced areas of increased signal intensity on MRI scans. We failed to find an association between the presence of hyperintensities and tumors $\left(\chi^{2}=.09\right)$ in this group of children (NF alone and NF plus brain tumor), which suggests that subjects with hyperintensities did not have a higher incidence of brain tumors than subjects without hyperintensities.

The results of this study using a large population of children and adolescents with NF generally support the earlier findings of Moore and colleagues (Moore et al., 1994) that children with NF are at risk for deficits in academic skills, memory, visual-spatial abilities, and concentration, but these difficulties are not significantly impacted by the additional diagnosis of brain tumor. Therefore, it is important to monitor children with NF with comprehensive neuropsychological assessments in addition to medical evaluations. The additional diagnosis of brain tumor does not increase the neuropsychological morbidity of NF unless the treatment includes cranial irradiation. It is suggested that future studies involving the neuropsychological assessment of children and adolescents with NF type I should include those patients diagnosed with a brain tumor, but that those treated with cranial irradiation should be eliminated from statistical analyses.

\section{References}

Beery, K., and Butenika, N. (1967) Developmental Test of Visual-Motor Integration. Chicago, III.: Follett Education Co.

Brookshire, B., Copeland, D.R., Moore, B.D., and Ater, J.L. (1990) Pretreatment neuropsychological status and associated factors in children with primary brain tumors. Neurosurgery 27, 887-891.

Buschke, H., and Fuld, P.A. (1974) Evaluating storage, retention, and retrieval in disordered memory and learning. Neurology 24, 1019-1025.

Cohen, B.H., Kaplan, A.M., and Packer, R.J. (1990-91) Management of intracranial neoplasms in children with neurofibromatosis type 1 and 2 . Pediatr. Neurosurg. 16, 66-72.

Cohen, M.E., Duffner, P.K., Kuhn, J.P., and Seidel, F.G. (1986) Neuroimaging in neurofibromatosis. Ann. Neurol. 20, 444.

Copeland, D.R., and Moore, B.D. (1992) Neuropsychological outcome among children treated at M.D. Anderson Cancer Center. Can. Bull. 44, 509-517.

Copeland, D.R., Fletcher, J.M., Pfefferbaum-Levine, B., Jaffe, N., Ried, H., and Maor, M. (1985) Neuropsychological sequelae of childhood cancer in long-term survivors. Pediatrics 75, 745-753.

Copeland, D.R., Dowell, R.E., Jr., Fletcher, J.M., Bordeaux, J.D., Sullivan, M.P., Jaffe, N., Frankel, L.S., Ried, H.L., and Cangir, A. (1988) Neuropsychological effects of childhood cancer treatment. J. Child. Neurol. 3, 53-62.

Crossen, J.R., Garwood, D., Glatstein, E., and Neuwelt, E.A. (1994) Neurobehavioral sequelae of cranial irradiation in adults: $A$ review of radiationinduced encephalopathy. J. Clin. Oncol. 12, 627-642.

Denckla, M.B., and Rudel, R. (1974) Rapid "automatized" naming of pictured objects, colors, letters, and numbers by normal children. Cortex 10 , 186-202.

Denckla, M.B., Hofman, K., Mazzocco, M.M., Melhem, E., Reiss, A.L., Bryan, R.N., Harris, E.L., Lee, J., Cox, C.S., and Schuerholz, L.J. (1996) Relationship between T2-weighted hyperintensities (unidentified bright objects) and lower IQs in children with neurofibromatosis-1. Am. J. Med. Genet. 67, 98-102.

Duffner, P.K., Cohen, M.E., Seidel, F.G., and Shucard, D.W. (1989) The signi- 
ficance of MRI abnormalities in children with neurofibromatosis. Neurology 39, 373-378.

Eldridge, R., Denckla, M.B., Bien, E., Myers, S., Kaiser-Kupfer, M.I., Pikus, A., Schlesinger, S.L., Parry, D.M., Dambrosia, J.M., Zasloff, M.A., Mulvihill, J.J. (1989) Neurofibromatosis type 1 (Recklinghausen's disease): Neurologic and cognitive assessment with sibling controls. Am. J. Dis. Child. $143,833-837$.

Eliason, M.J. (1986) Neurofibromatosis: Implications for learning and behavior. J. Dev. Behav. Pediatr. 7, 175-179.

Ferner, R.E., Chaudhuri, R., Bingham, J., Cos, T., and Hughes, R.A.C. (1993) $M R I$ in neurofibromatosis 1: The nature and evolution of increased intensity T2 weighted lesions and their relationship to intellectual impairment. J. Neurol. Neurosurg. Psychiatry 56, 492-495.

Fletcher, J.M. (1985) Memory for verbal and nonverbal stimuli in learning disabled subgroups: Analysis by selective reminding. J. Exp. Child Psychol. 40, 244-259.

Fletcher, J.M., and Copeland, D.R. (1988) Neurobehavioral effects of central nervous system prophylactic treatment of cancer in children. J. Clin. Exp. Neuropsychol. 10, 495-537.

Gaddes, W.H., and Crockett, D.J. (1975) The Spreen-Benton aphasia tests: Normative data as a measure of normal language development. Brain Lang. 2, 257-280.

Hofman, K.J., Harris, E.L., Bryan, R.N., and Denckla, M.B. (1994) Neurofibromatosis type 1: The cognitive phenotype. J. Pediatr. 124, S1-S8.

Hollingshead, A.B. (1975) Four Factor Index of Social Status. New Haven, Conn.: Yale University.

Jastak, S.R., and Wilkinson, G.S. (1984) WRAT-R: Wide Range Achievement Test-Revised Administration Manual. Wilmington, Del.: Jastak Associates, Inc.

Joy, P., Roberts, C., North, K., and de Silva, M. (1995) Neuropsychological function and MRI abnormalities in neurofibromatosis type I. Dev. Med. Child Neurol. 37, 906-914.

Legius, E., Descheemaeker, M.J., Spaepen, A., Casaer, P., and Fryns, J.P. (1994) Neurofibromatosis type 1 in childhood: A study of the neuropsychological profile in 45 children. Genet. Counseling 5, 51-60.

Leiner, H.C., Leiner, A.L., and Dow, R.S. (1991) The human cerebro-cerebellar system: Its computing, cognitive, and language skills. Behav. Brain Res. 44, 113-128.

Lindgren, S.D., and Benton, A.L. (1980) Developmental patterns of visuospatial judgment. J. Pediatr. Psychol. 5, 217-225.

Listernick, R., Charrow, J., Greenwald, M.J., and Easterly, N.B. (1989) Optic gliomas in children with neurofibromatosis type 1. J. Pediatr. 114, 788-792.

Listernick, R., Charrow, J., and Greenwald, M. (1992) Emergence of optic pathway gliomas in children with neurofibromatosis type 1 after normal neuroimaging results. J. Pediatr. 121, 584-587.

Listernick, R., Charrow, J., Greenwald, M., and Mets, M. (1994) Natural history of optic pathway tumors in children with neurofibromatosis type 1: A longitudinal study. J. Pediatr. 125, 63-66.

Mazzocco, M.M., Turner, J.E., Denckla, M.B., Hofman, K.J., Scanlon, D.C., and Vellutino, F.R. (1997) Language and reading deficits associated with neurofibromatosis type 1: Evidence for a not-so-nonverbal learning disability. Dev. Neuropsychol. 11, 504-522.

McCarthy, D. (1972) McCarthy Scales of Children's Abilities. New York, N.Y.: The Psychological Corporation.

Moore, B.D., III, Ater, J.L., Needle, M.N., Slopis, J., and Copeland, D.R. (1994) Neuropsychological profile of children with neurofibromatosis, brain tumor, or both. J. Child Neurol. 9, 368-377.

Moore, B.D., Slopis, J.M., Schomer, D., Jackson, E.F., and Levy, B.M. (1996) Neuropsychological significance of areas of high signal intensity on brain MRIs of children with neurofibromatosis. Neurology 46, 1660-1668.

Mulvihill, J.J., Parry, D.M., Sherman, J.L., Pikus, A., Kaiser-Kupfer, M.I., and Eldridge, R. (1990) NIH conference. Neurofibromatosis 1 (Recklinghausen disease) and neurofibromatosis 2 (bilateral acoustic neurofibromatosis): An update. Ann. Internal Med. 113, 39-52.

$\mathrm{NIH}$ (National Institutes of Health) (1988) Neurofibromatosis conference statement. National Institutes of Health consensus Development Conference. Arch. Neurol. 45, 575-578.

North, K., Joy, P., Yuille, D., Cocks, N., Mobbs, E., Hutchins, P., McHugh, K., and de Silva, M. (1994) Specific learning disability in children with neurofibromatosis type 1: Significance of MRI abnormalities. Neurology 44, 878-883.

North, K.N., Riccardi, V., Samango-Sprouse, C., Ferner, R., Moore, B., Legius, E., Ratner, N., and Denckla, M.B. (1997) Cognitive function and academic performance in neurofibromatosis 1: Consensus statement from the NF1 Cognitive Disorders Task Force. Neurology 48, 1121-1127.

Packer, R.J., Meadows, A.T., Rorke, L.B., Goldwein, J.L., and D'Angio, G. (1987) Long-term sequelae of cancer treatment on the central nervous system in childhood. Med. Pediatr. Oncol. 15, 241-253.

Reitan, R. (1969) Manual for Administration of Neuropsychological Test Batteries on Adults and Children. Bloomington, Ind.: Indiana University Press.

Riccardi, V.M. (1981) Von Recklinghausen neurofibromatosis. N. Engl. J. Med. 305, 1617-1627.

Riccardi, V.M. (1982) The multiple forms of neurofibromatosis. Pediatr. Rev. 3, 293-298.

Ris, M.D., and Noll, R.B. (1994) Long-term neurobehavioral outcome in pediatric brain-tumor patients: Review and methodological critique. J. Clin. Exp. Neuropsychol. 16, 21-42.

Roos, K.L., and Dunn, D.W. (1992) Neurofibromatoses. CA Cancer J. Clin. 42, 241-254.

Rosner, J., and Simon, D.P. (1971) The Auditory Analysis Test: An initial report. J. Learn. Dis. 7, 40-47.

Satz, P., and Fletcher, J.M. (1982) The Florida Kindergarten Screening Battery. Odessa, Fla.: Psychological Assessment Resources, Inc.

Stine, S.B., and Adams, W.V. (1989) Learning problems in neurofibromatosis patients. Clin. Orthop. 245, 43-48.

Thorndike, R.L., Hagen, E.P., and Sattler, J.M. (1986) Stanford-Binet Intelligence Scale: Guide for Administering and Scoring the Fourth Edition. Fourth edition. Chicago: The Riverside Publishing Company.

Varnhagen, C.K., Lewin, S., Das, J.P., Bowen, P., Ma, K., and Klimek, M. (1988) Neurofibromatosis and psychological processes. J. Dev. Behav. Pediatr. 9, 257-265.

Wadsby, M., Lindehammar, H., and Eeg-Olofsson, O. (1989) Neurofibromatosis in childhood: Neuropsychological aspects. Neurofibromatosis 2, 251-260.

Wechsler, D. (1974) WISC-R, Manual for the Wechsler Intelligence Scale for Children-Revised. New York, N.Y.: The Psychological Corporation.

Wechsler, D. (1991) Wechsler Intelligence Scale for Children. Third edition. New York, N.Y.: Harcourt, Brace, Jovancovich, Inc.,

Woodcock, R.W., Johnson, M.B., and Mather, N. (1989) Woodcock Johnson Psycho-Educational Battery-Revised. Allen, Texas: DLM Teaching Resources.

Wright, T.L., and Bresnan, M.J. (1976) Radiation-induced cerebrovascular disease in children. Neurology 26, 540-543. 